\title{
Quaternary Structure Transitions of Human Hemoglobin: An Atomic-Level View of the Functional Intermediate States
}

Nicole Balasco ${ }^{\mathrm{a}}$, Josephine Alba ${ }^{\mathrm{b}, 1}$, Marco D’Abramo ${ }^{\mathrm{b}, *}$, and Luigi Vitagliano ${ }^{\mathrm{a},{ }^{*}}$

${ }^{a}$ Institute of Biostructures and Bioimaging, CNR, Via Mezzocannone 16, 80134 Naples, Italy.

bDepartment of Chemistry, University of Rome Sapienza, P.le A.Moro 5, 00185 Rome, Italy.

*Correspondence: luigi.vitagliano@unina.it (L.V.); marco.dabramo@uniroma1.it (M.D.).

${ }^{1}$ Current address: Université de Fribourg 


\section{Supporting Information}
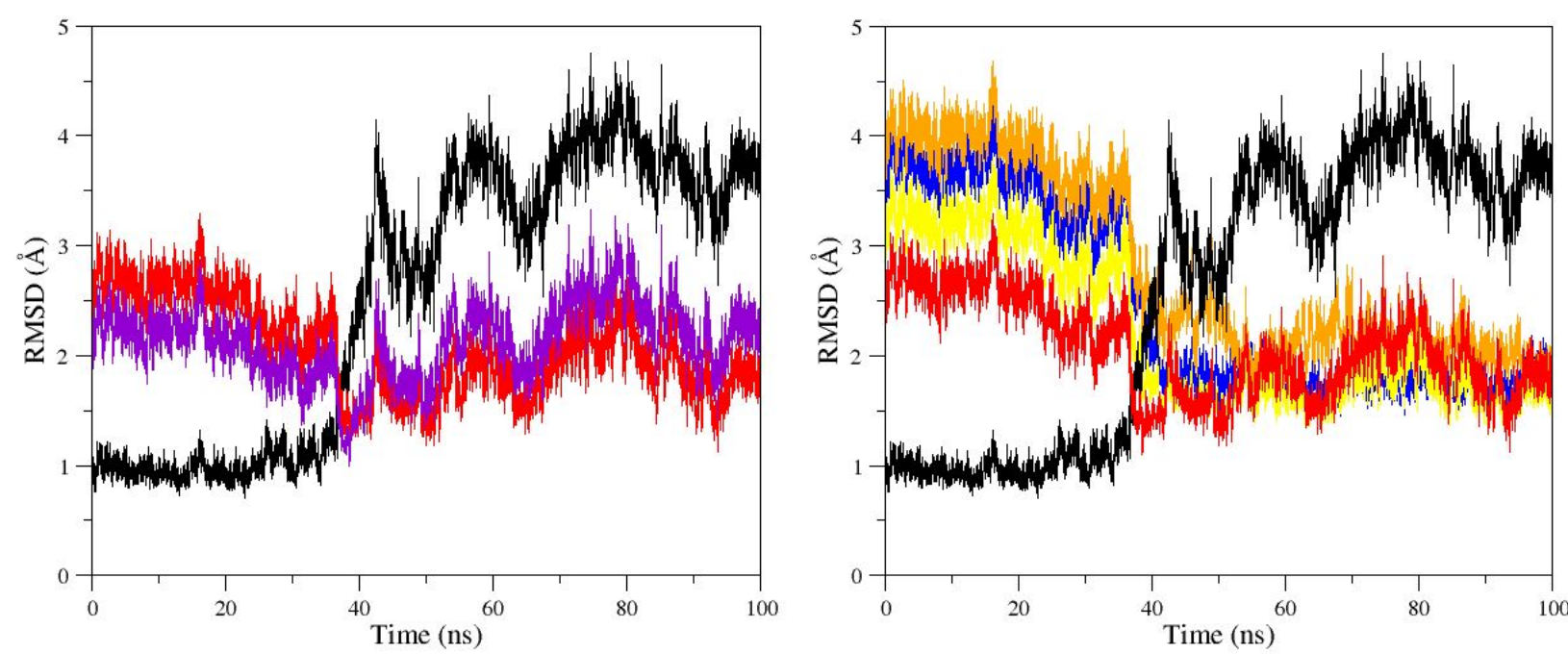

(a)

(b)
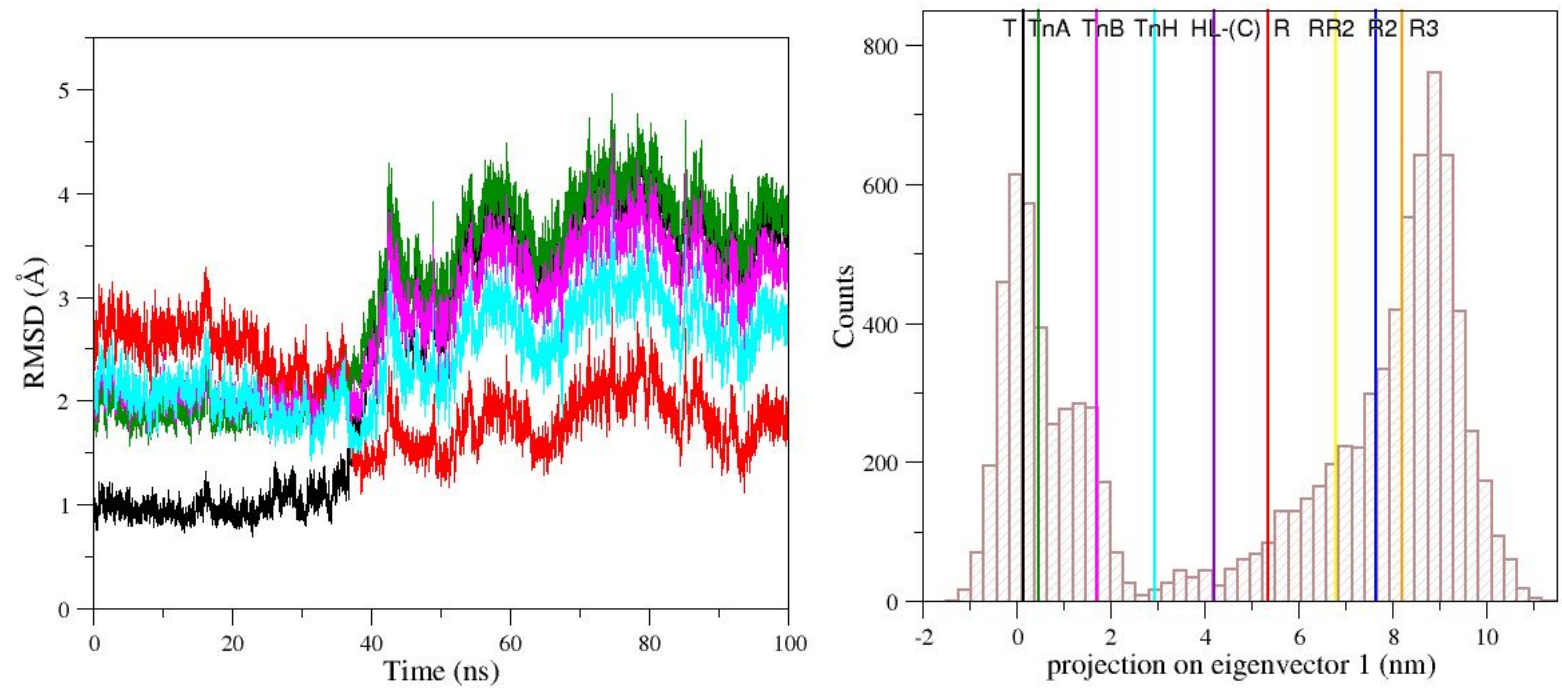

(c)

(d)

Figure S1. (a) RMSD values (computed on the $\mathrm{C}^{\alpha}$ atoms) of the $\mathrm{T} 0 \mathrm{~b}$ trajectory structures versus the starting T model (black, PDB ID: 2DN2), the R state (red, PDB ID: 2DN1), and the intermediate HL(C) state (violet, PDB ID: 4N7P). (b) RMSD values computed against the off-pathway structures R2 (blue, PDB ID: 1BBB), RR2 (yellow, PDB ID: 1MKO) and R3 (orange, PDB ID: 4NI0). (c) RMSD values computed against the intermediate states identified for HbTn: TnA (green, PDB ID: 5LFG), TnB (magenta, PDB ID: 5LFG) and TnH (cyan, PDB ID: 3D1K). RMSD values refer to the productive run without the equilibration steps producing the initial drift. (d) Projection on the first eigenvector of the T0b trajectory structures. The vertical solid lines correspond to the projections of the crystallographic structures of human HbA states: T (black, PDB ID: 2DN2), R (red, PDB ID: 2DN1), intermediate HL-(C) (violet, PDB ID: 4N7P), R2 (blue, PDB ID: 1BBB), RR2 (yellow, PDB ID: $1 \mathrm{MKO}$ ) and R3 (orange, PDB ID: 4NI0) and of the HbTn intermediates: TnA (green, PDB ID: 5LFG), TnB (magenta, PDB ID: 5LFG) and TnH (cyan, PDB ID: 3D1K). 


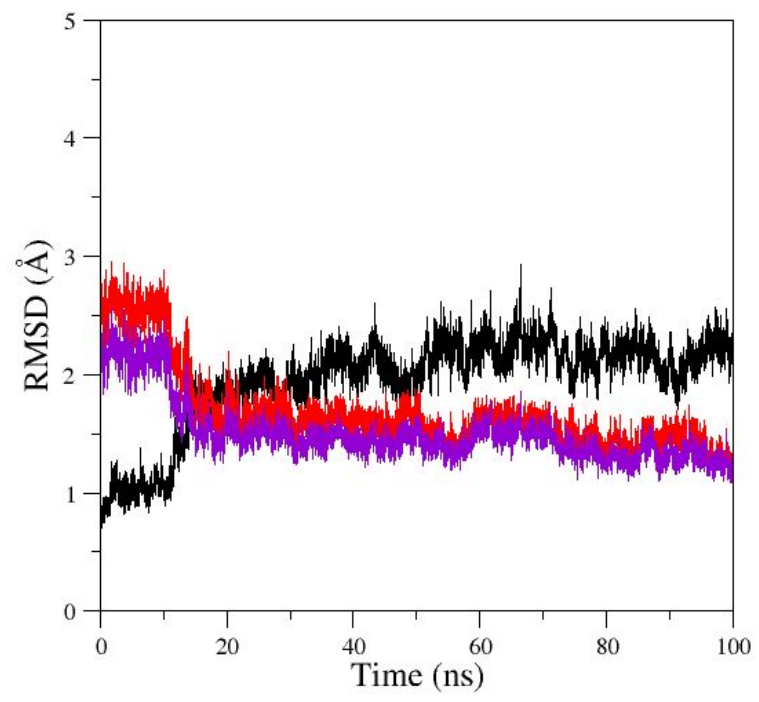

(a)

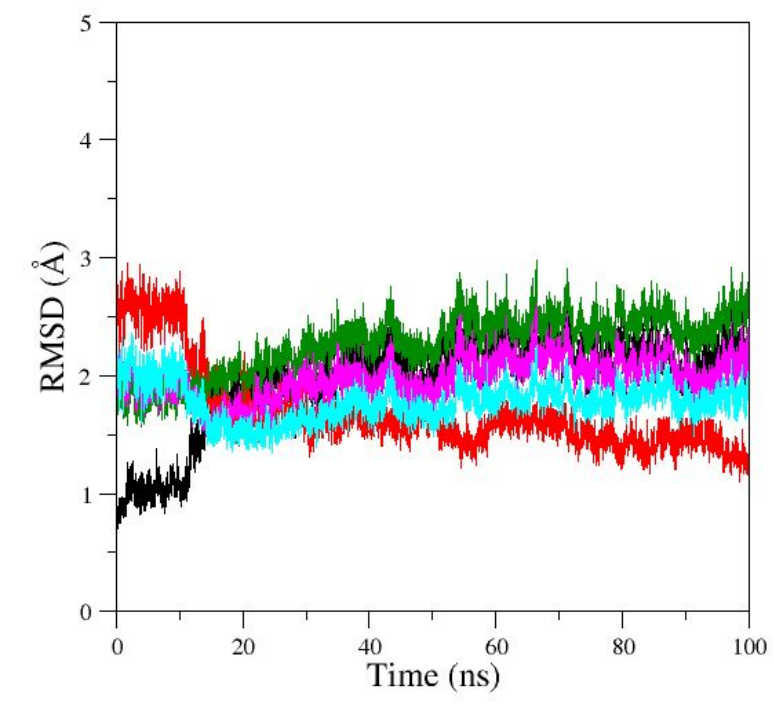

(c)

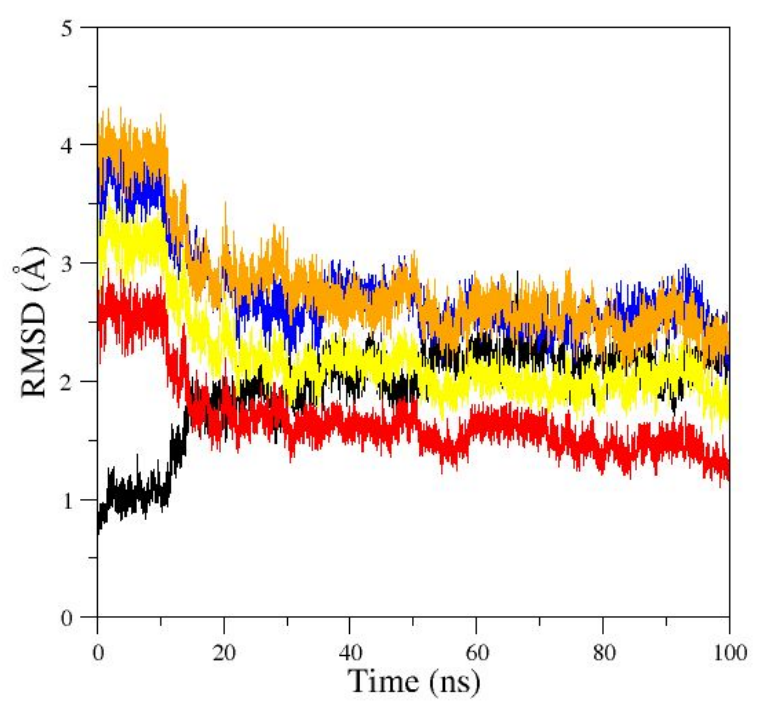

(b)

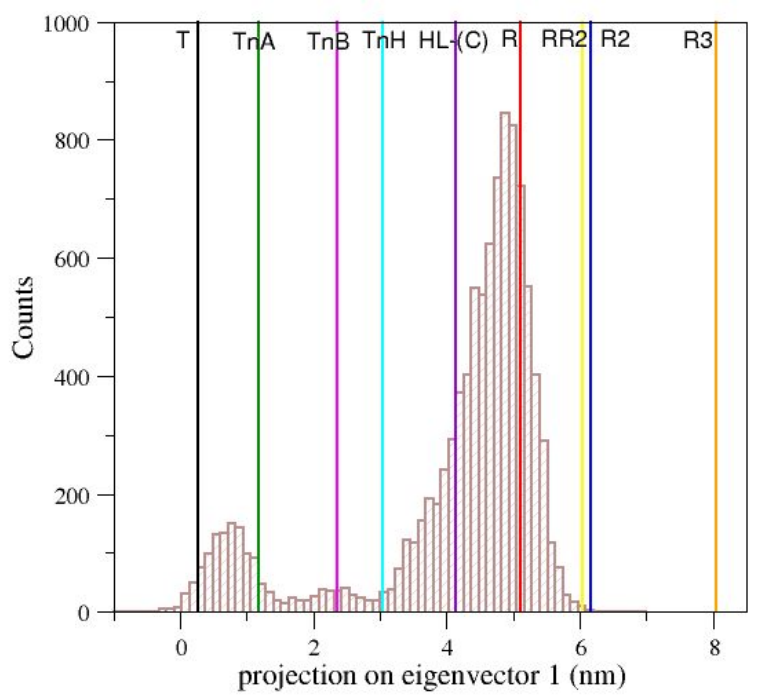

(d)

Figure S2. (a) RMSD values (computed on the $\mathrm{C}^{\alpha}$ atoms) of the $\mathrm{T} 0 \mathrm{c}$ trajectory structures versus the starting T model (black, PDB ID: 2DN2), the R state (red, PDB ID: 2DN1), and the intermediate HL(C) state (violet, PDB ID: 4N7P). (b) RMSD values computed against the off-pathway structures R2 (blue, PDB ID: 1BBB), RR2 (yellow, PDB ID: 1MKO) and R3 (orange, PDB ID: 4NI0). (c) RMSD values computed against the intermediate states identified for HbTn: TnA (green, PDB ID: 5LFG), TnB (magenta, PDB ID: 5LFG) and TnH (cyan, PDB ID: 3D1K). RMSD values refer to the productive run without the equilibration steps producing the initial drift. (d) Projection on the first eigenvector of the T0c trajectory structures. The vertical solid lines correspond to the projections of the crystallographic structures of human HbA states: T (black, PDB ID: 2DN2), R (red, PDB ID: 2DN1), intermediate HL-(C) (violet, PDB ID: 4N7P), R2 (blue, PDB ID: 1BBB), RR2 (yellow, PDB ID: 1MKO) and R3 (orange, PDB ID: 4NI0) and of the HbTn intermediates: TnA (green, PDB ID: 5LFG), TnB (magenta, PDB ID: 5LFG) and TnH (cyan, PDB ID: 3D1K). 


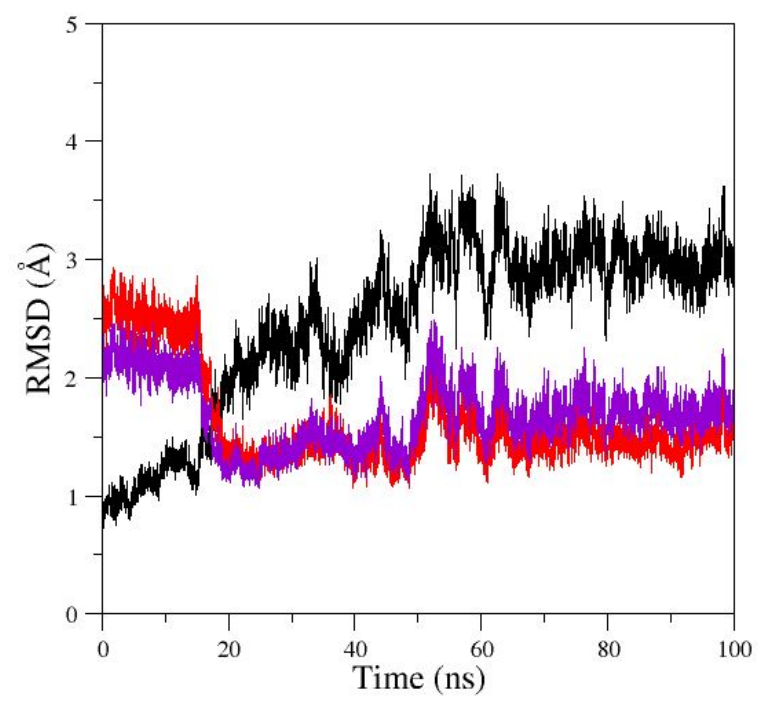

(a)

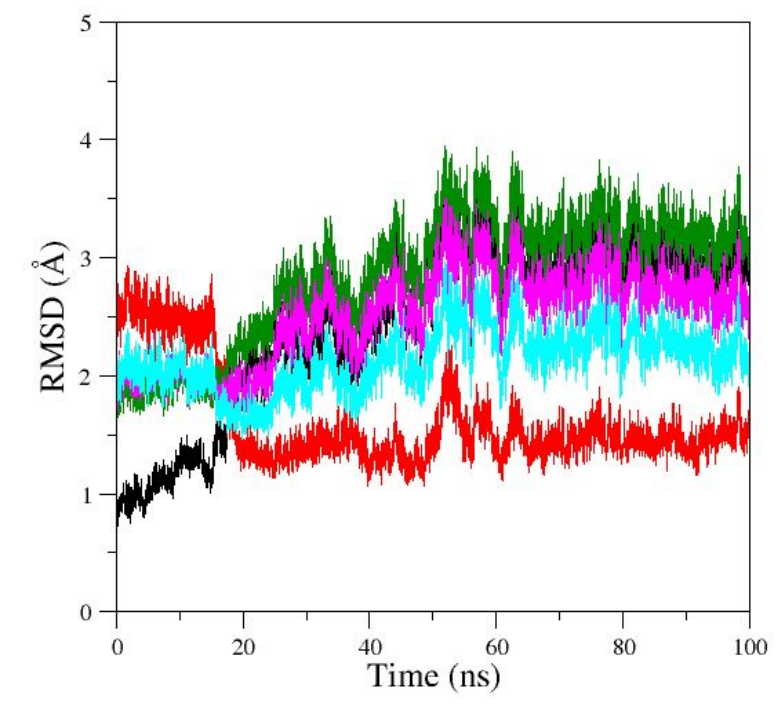

(c)

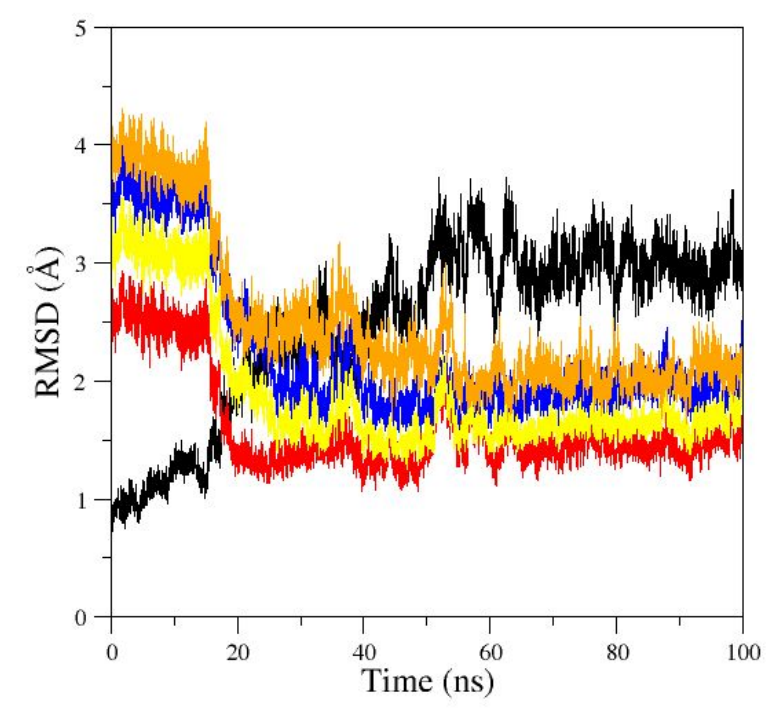

(b)

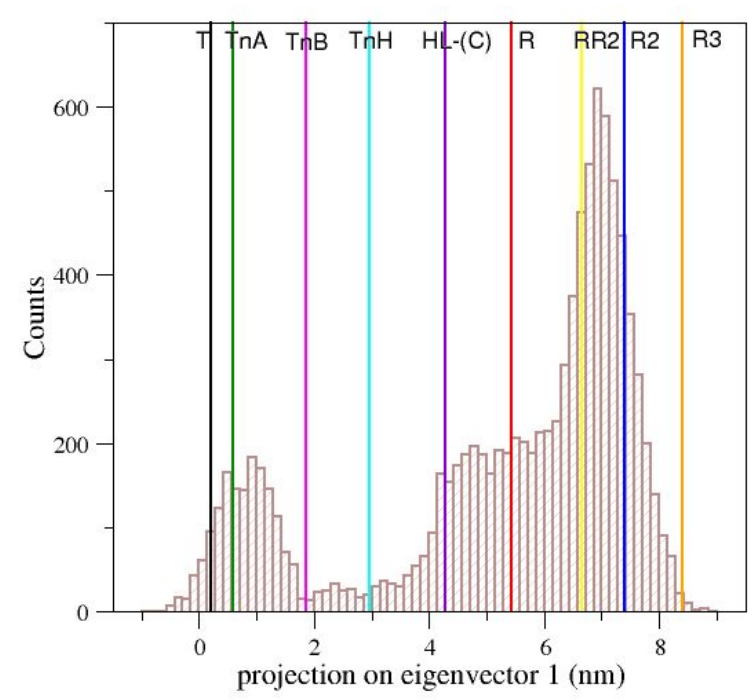

(d)

Figure S3. (a) RMSD values (computed on the $\mathrm{C}^{\alpha}$ atoms) of the T0d trajectory structures versus the starting T model (black, PDB ID: 2DN2), the R state (red, PDB ID: 2DN1), and the intermediate HL(C) state (violet, PDB ID: 4N7P). (b) RMSD values computed against the off-pathway structures R2 (blue, PDB ID: 1BBB), RR2 (yellow, PDB ID: 1MKO) and R3 (orange, PDB ID: 4NI0). (c) RMSD values computed against the intermediate states identified for HbTn: TnA (green, PDB ID: 5LFG), TnB (magenta, PDB ID: 5LFG) and TnH (cyan, PDB ID: 3D1K). RMSD values refer to the productive run without the equilibration steps producing the initial drift. (d) Projection on the first eigenvector of the T0d trajectory structures. The vertical solid lines correspond to the projections of the crystallographic structures of human HbA states: T (black, PDB ID: 2DN2), R (red, PDB ID: 2DN1), intermediate HL-(C) (violet, PDB ID: 4N7P), R2 (blue, PDB ID: 1BBB), RR2 (yellow, PDB ID: 1MKO) and R3 (orange, PDB ID: 4NI0) and of the HbTn intermediates: TnA (green, PDB ID: 5LFG), TnB (magenta, PDB ID: 5LFG) and TnH (cyan, PDB ID: 3D1K). 


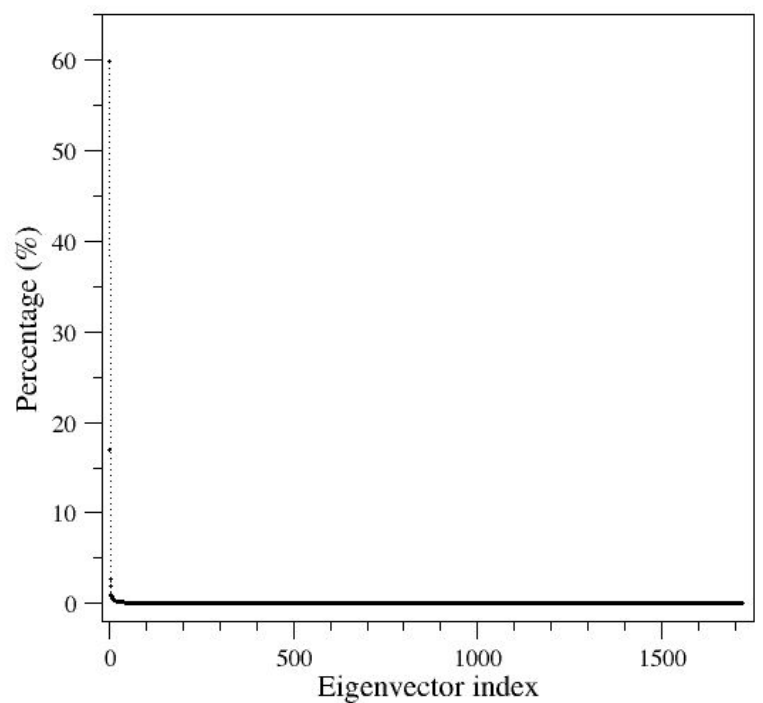

(a)

Figure S4. (a) Spectrum of the eigenvalues (as percentage) derived from the covariance matrix diagonalization of the T0 trajectory. (b) An enlargement showing only the first 20 eigenvalues is reported.

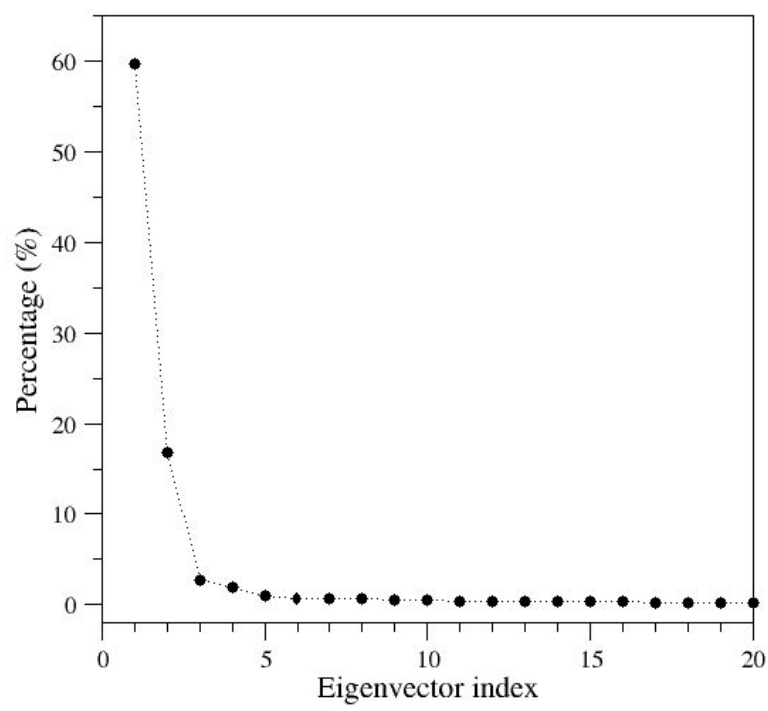

(b)

\section{trix}



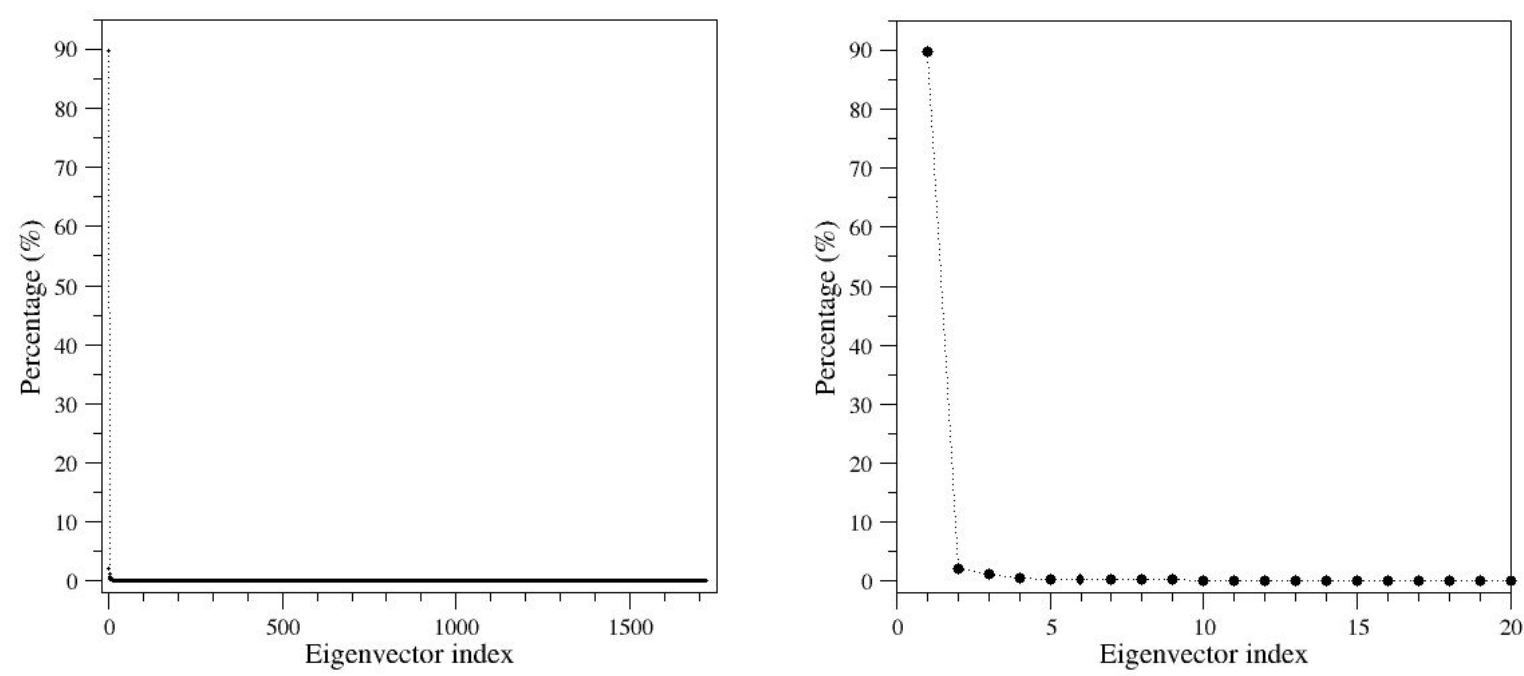

(a)
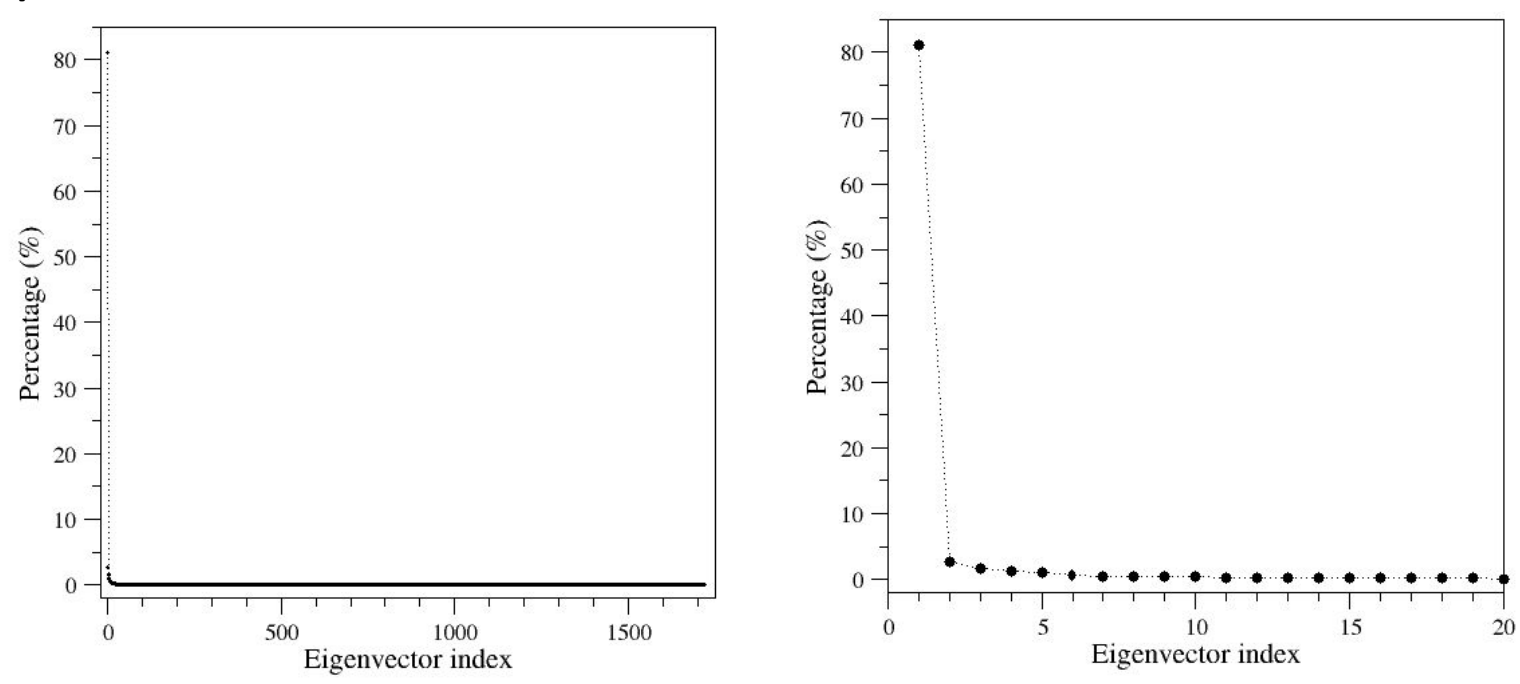

(b)
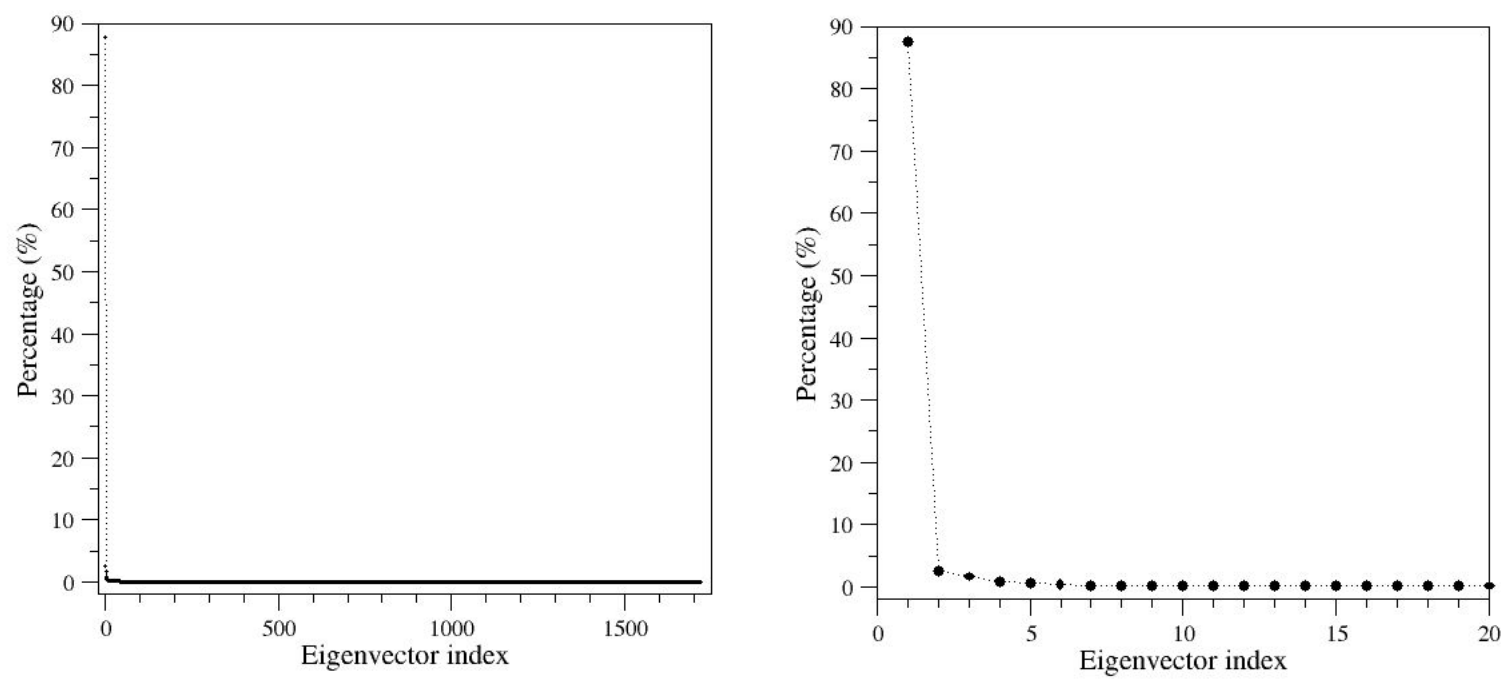

(c)

Figure S5. Spectrum of the eigenvalues (as percentage) derived from the covariance matrix diagonalization of the T0b (a), T0c (b), T0d (c) trajectories. An enlargement showing only the first 20 eigenvalues is reported on the right side of each plot. 

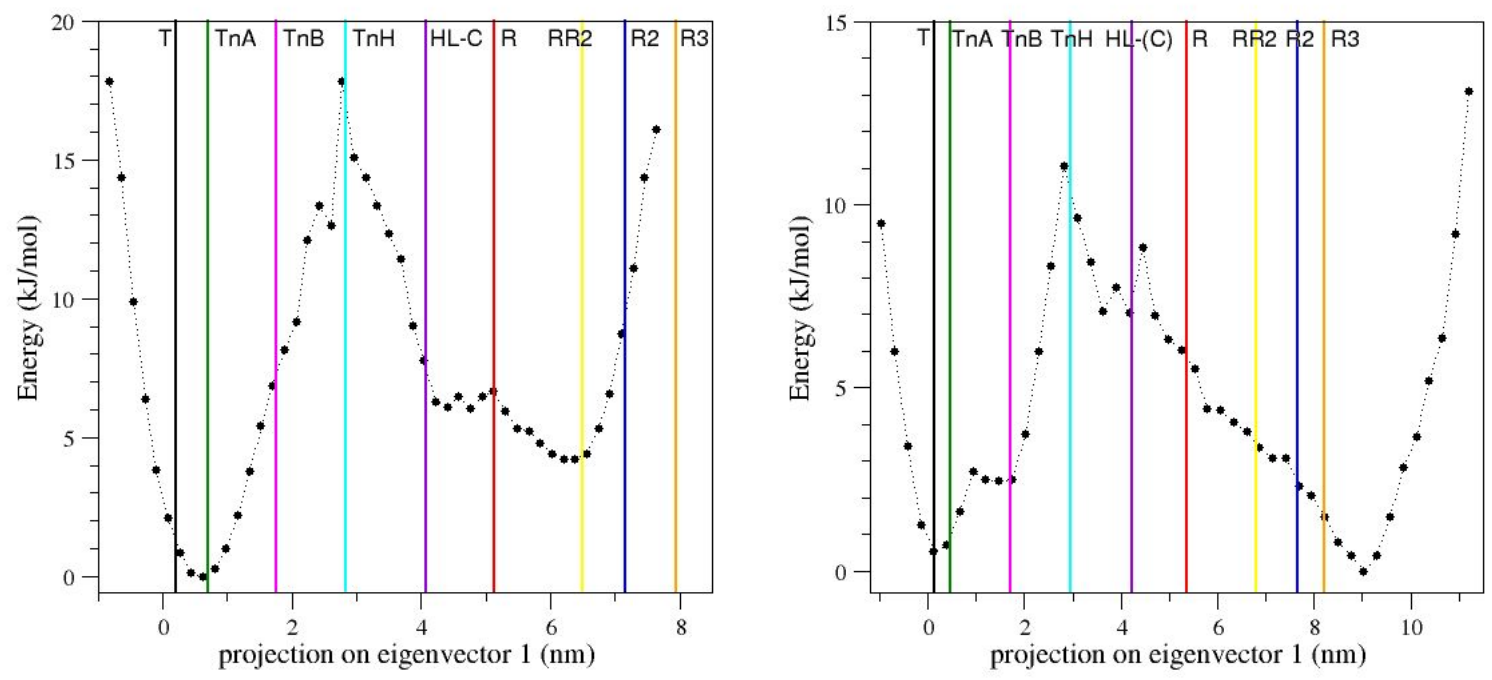

(a)

(b)
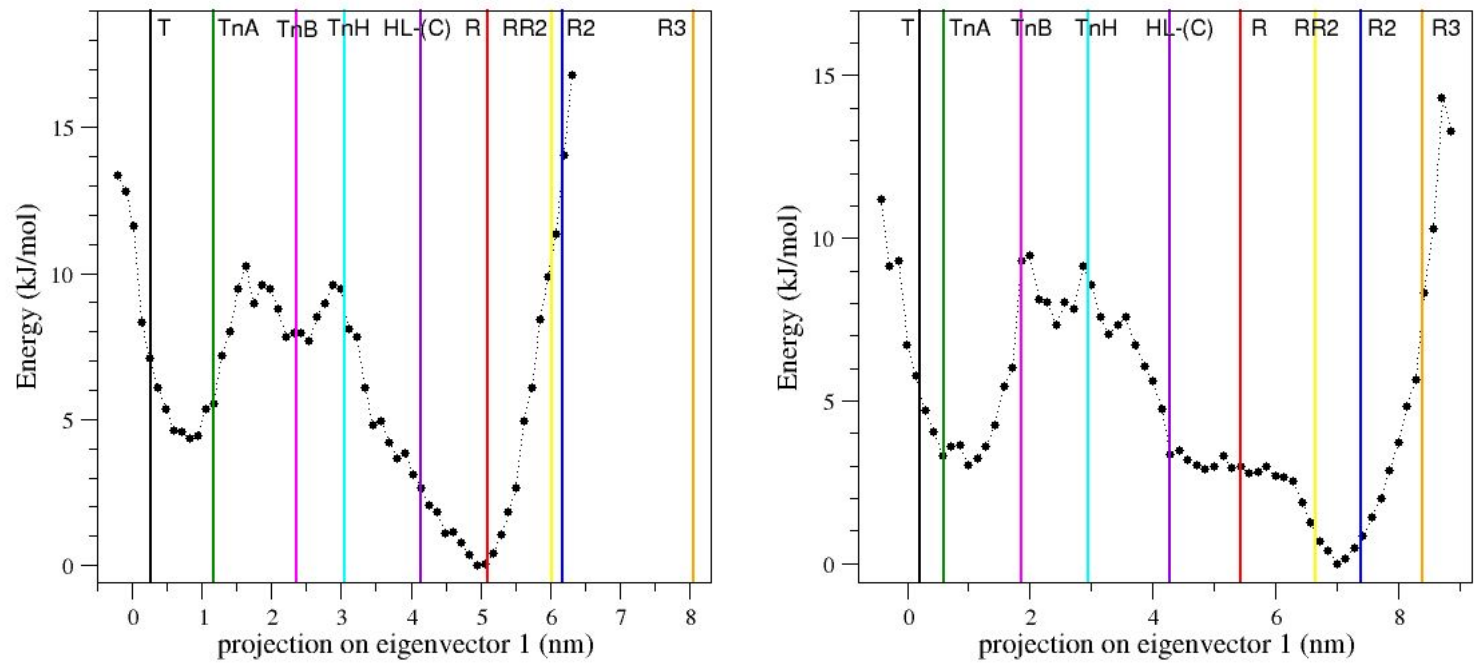

(c)

(d)

Figure S6. Estimation of the relative free energy of the different structural states detected in the T-R transition of the T0 (a), T0b (b), T0c (c), and T0d (d) MD simulations. The projection was preliminary dissected in a discrete number of states $(i)$ whose populations were calculated. The black points represent the free energies as obtained by $\Delta \mathrm{G}=-\mathrm{RT} \ln \left(\mathrm{p}_{i} / \mathrm{p}_{\text {ref }}\right)$, where $\mathrm{p}_{i}$ is the population of the structures whose projection fall in the $i$ state and $\mathrm{p}_{\text {ref }}$ is the population of the most populated state, $\mathrm{R}$ is the gas constant, $T$ is the temperature of the system, and $\Delta \mathrm{G}$ is the free energy difference between the two states. 

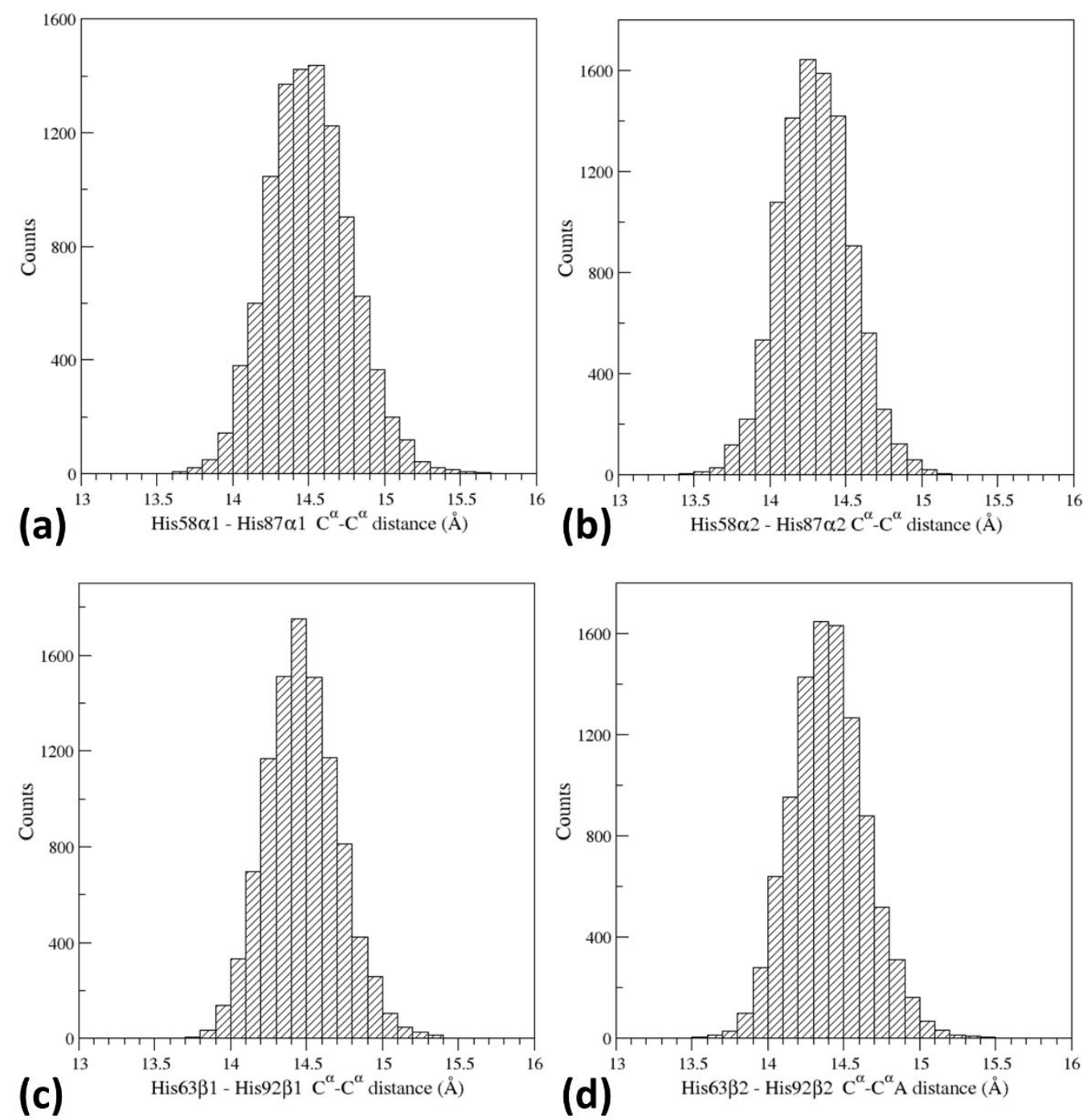

Figure S7. Distributions in the T0 trajectory structures of the distance between the $\mathrm{C}^{\alpha}$ atoms of the distal and proximal His in the (a) $\alpha 1$, (b) $\alpha 2$, (c) $\beta 1$ and (d) $\beta 2$ chains. 

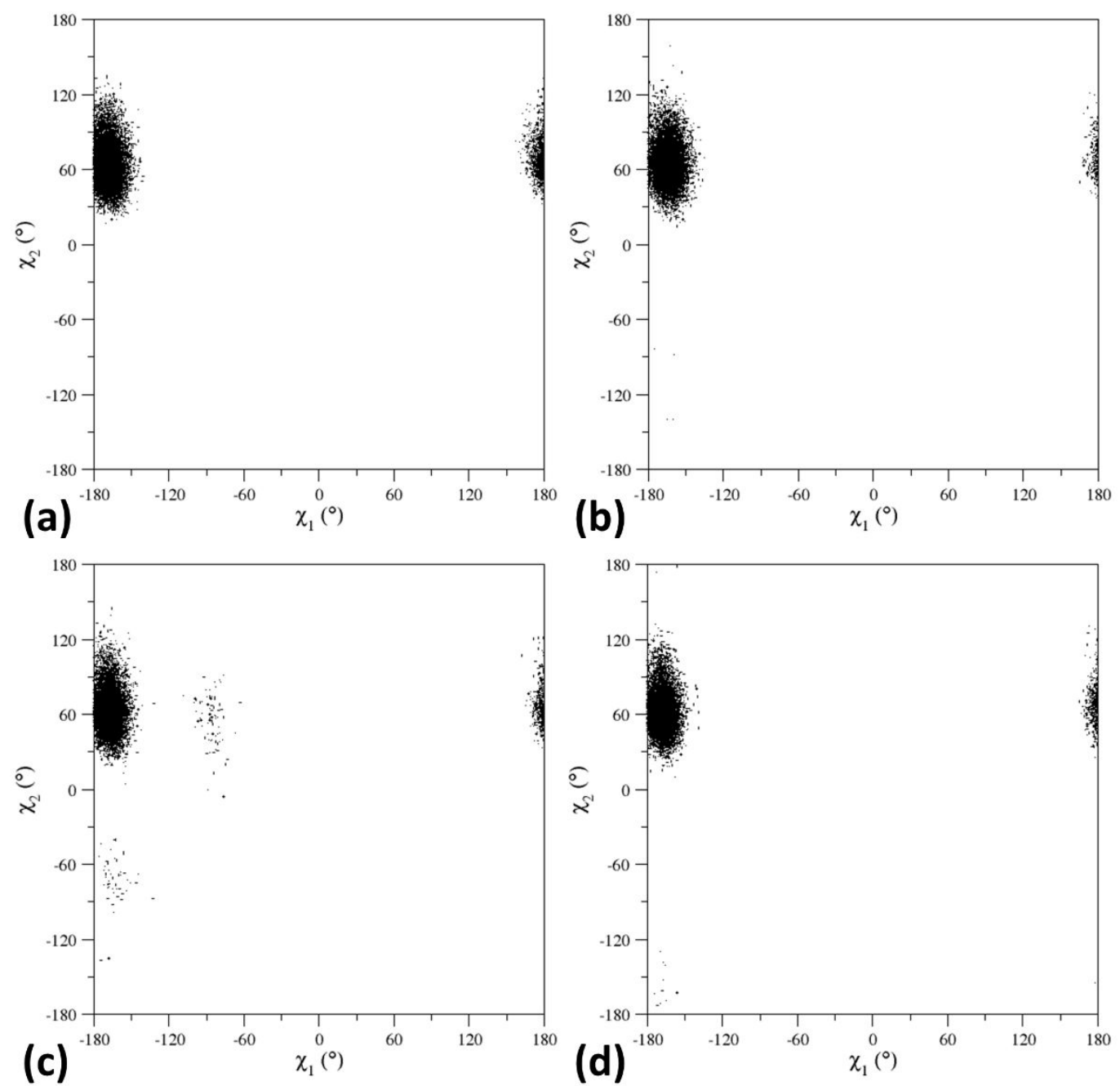

Figure S8. Distributions in the T0 trajectory structures of the $\chi_{1}$ and $\chi_{2}$ dihedral angle of the distal His of the (a) $\alpha 1$, (b) $\alpha 2$, (c) $\beta 1$ and (d) $\beta 2$ chains. 

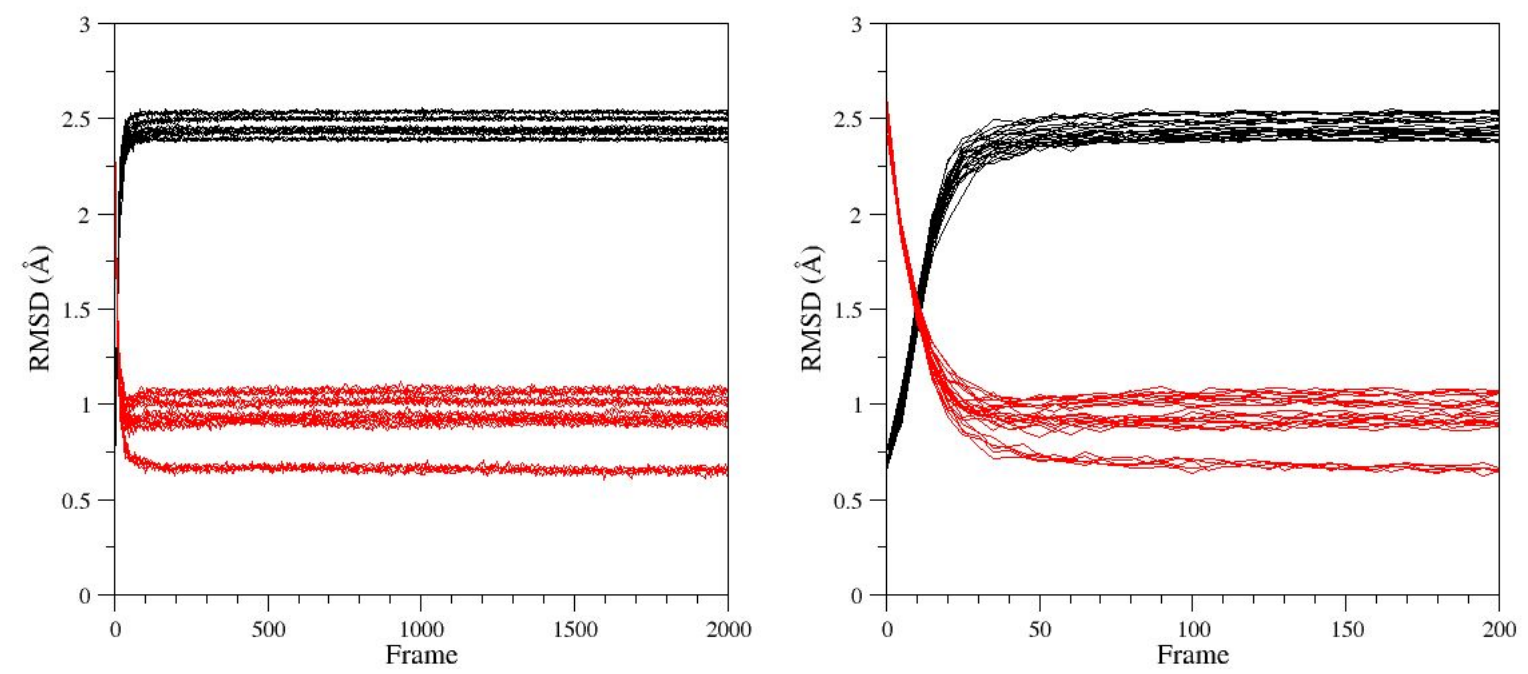

(a)
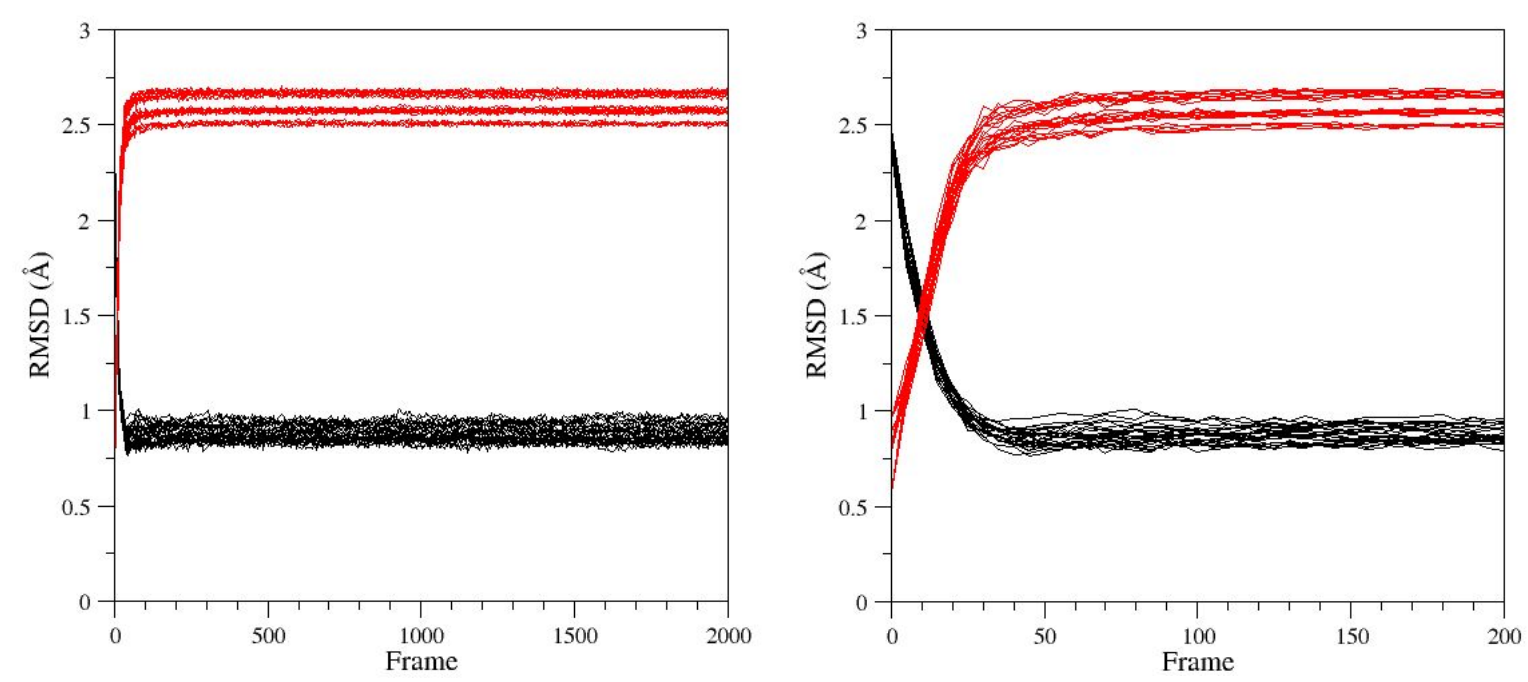

(b)

Figure S9. RMSD values, computed on the $\mathrm{C}^{\alpha}$ atoms, of trajectory structures versus the crystallographic T (black, PDB ID: 2DN2) and R (red, PDB ID: 2DN1) states of HbA in the (a) T-R and (b) R-T EDS. A snapshot of the RMSD values including the first 200 frames is reported on the right side. 
Table S1. Details of representative crystallographic structures of $\mathrm{HbA}$ and $\mathrm{HbTn}$.

\begin{tabular}{|c|c|c|c|c|c|}
\hline Hb State & $\begin{array}{l}\text { PDB } \\
\text { code }\end{array}$ & $\begin{array}{c}\text { Resol. } \\
(\AA)\end{array}$ & R/Rfree & Asymmetric unit & Binding state \\
\hline $\mathrm{T}$ & 2DN2 & 1.25 & $0.179 /$ null & tetramer & Unliganded \\
\hline $\mathrm{R}$ & 2DN1 & 1.25 & $0.195 /$ null & dimer & Fully-liganded $\left(\mathrm{O}_{2}\right)$ \\
\hline $\mathrm{R} 2$ & $1 \mathrm{BBB}$ & 1.7 & $0.184 /$ null & tetramer & Fully-liganded (CO) \\
\hline RR2 & $1 \mathrm{MKO}$ & 2.18 & $0.200 / 0.284$ & tetramer & Fully-liganded (CO) \\
\hline R3 & $4 \mathrm{NIO}$ & 2.15 & $0.228 / 0.259$ & dimer & Fully-liganded (CO) \\
\hline HL-(C) & 4N7P & 2.8 & $0.259 / 0.287$ & $\begin{array}{l}3 \text { tetramers: HL-(A), } \\
\text { HL-(B), HL-(C) }\end{array}$ & Half-liganded (CO) \\
\hline $\mathrm{TnT}$ & $3 \mathrm{NFE}$ & 2.01 & $0.185 / 0.250$ & tetramer & Unliganded \\
\hline $\mathrm{TnR}$ & $1 \mathrm{~T} 1 \mathrm{~N}$ & 2.20 & $0.192 /$ null & dimer & Fully-liganded (CO) \\
\hline $\operatorname{Tn} A, \operatorname{TnB}$ & $5 \mathrm{LFG}$ & 1.94 & $0.181 / 0.245$ & 2 dimers: $\operatorname{Tn} A, \operatorname{Tn} B$ & Fully-liganded (CO) \\
\hline $\mathrm{TnH}$ & $3 \mathrm{D} 1 \mathrm{~K}$ & 1.25 & $0.167 / 0.202$ & dimer & Half-liganded (CO) \\
\hline
\end{tabular}


Table S2. Local descriptors of the structural state of representative crystallographic structures of $\mathrm{HbA}$ and $\mathrm{HbTn}$.

\begin{tabular}{|c|c|c|c|c|c|}
\hline Hb State & $\begin{array}{l}\text { PDB } \\
\text { code }\end{array}$ & 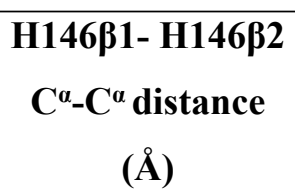 & 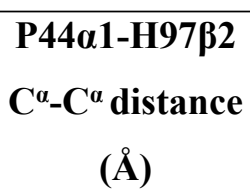 & 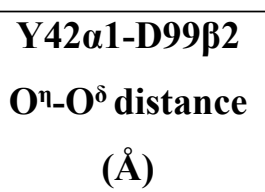 & 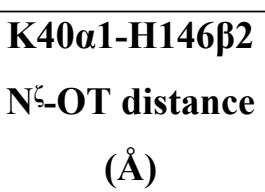 \\
\hline $\mathrm{T}$ & 2DN2 & 30.9 & 7.3 & 2.5 & 2.8 \\
\hline $\mathrm{R}$ & 2DN1 & 12.5 & 12.6 & 8.0 & 15.8 \\
\hline $\mathrm{R} 2$ & $1 \mathrm{BBB}$ & 8.0 & 14.8 & 9.4 & 21.2 \\
\hline RR2 & $1 \mathrm{MKO}$ & 12.4 & 13.8 & 9.0 & 17.6 \\
\hline R3 & $4 \mathrm{NIO}$ & 22.2 & 15.9 & 10.5 & 15.0 \\
\hline HL-(C) & 4N7P & 14.0 & 11.6 & 6.9 & 13.9 \\
\hline TnT & $3 \mathrm{NFE}$ & 32.0 & 7.5 & 3.1 & 2.7 \\
\hline TnR & $1 \mathrm{~T} 1 \mathrm{~N}$ & 19.2 & 12.6 & 7.6 & 11.6 \\
\hline TnA & $5 \mathrm{LFG}$ & 32.8 & 8.6 & 2.5 & 3.3 \\
\hline TnB & $5 \mathrm{LFG}$ & H146 missing & 12.1 & 3.3 & H146 missing \\
\hline TnH & $3 \mathrm{D} 1 \mathrm{~K}$ & 27.1 & 12.3 & 4.8 & 12.9 \\
\hline
\end{tabular}


Table S3. Structural features of the heme pocket in representative crystallographic structures of $\mathrm{HbA}$ and $\mathrm{HbTn}: \mathrm{C}^{\alpha}-\mathrm{C}^{\alpha}$ distance between proximal and distal His residues and rotameric state of the distal His side chain.

\begin{tabular}{|c|c|c|c|c|c|c|c|c|}
\hline \multirow[t]{2}{*}{ Hb State } & \multicolumn{4}{|c|}{$\begin{array}{c}\mathrm{C}^{\alpha}\left(\text { HisF8)-C }{ }^{\alpha}(\text { HisE7) }\right. \\
\text { distance }(\AA)\end{array}$} & \multicolumn{4}{|c|}{$\begin{array}{c}\text { Distal His sidechain } \\
\qquad 1 / \chi^{2}\end{array}$} \\
\hline & $\alpha 1$ & $\beta 1$ & $\alpha 2$ & $\beta 2$ & $\alpha 1$ & $\beta 1$ & $\alpha 2$ & $\beta 2$ \\
\hline $\mathrm{T}$ & 14.4 & 14.3 & 14.5 & 14.3 & $-171.9 / 61.8$ & $-173.7 / 66.6$ & $-173.0 / 64.3$ & $-170.7 / 58.2$ \\
\hline $\mathrm{R}$ & 14.2 & 14.4 & - & - & $-168.8 / 64.4$ & $-168.8 / 72.6$ & - & - \\
\hline R2 & 14.5 & 14.5 & 14.4 & 14.5 & $-153.9 / 71.8$ & $-164.4 / 78.1$ & $-150.4 / 67.1$ & $-162.5 / 67.2$ \\
\hline RR2 & 14.1 & 14.4 & 14.1 & 14.2 & $-152.4 / 75.0$ & $-174.2 / 80.1$ & $-151.5 / 74.4$ & $163.7 / 65.2$ \\
\hline R3 & 14.1 & 14.2 & - & - & $-150.2 / 71.0$ & $-159.0 /-63.5$ & - & - \\
\hline HL-(C) & 13.8 & 14.3 & 14.1 & 14.4 & $-178.7 / 60.4$ & $-157.9 / 55.8$ & $-175.1 / 28.4$ & $-151.1 / 66.5$ \\
\hline TnT & 13.4 & 14.3 & 13.1 & 14.1 & $175.1 /-109.2$ & $-164.5 / 64.2$ & $174.7 /-119.6$ & $-167.4 / 73.5$ \\
\hline TnR & 14.3 & 14.3 & - & - & $-162.7 / 78.4$ & $-154.6 / 90.6$ & - & - \\
\hline $\operatorname{TnA}$ & 14.0 & 13.1 & - & - & $-152.7 / 59.2$ & $-71.9 / 74.9$ & - & - \\
\hline TnB & 12.8 & 13.1 & - & - & $-81.7 / 104.0$ & $-71.4 / 80.5$ & - & - \\
\hline $\mathrm{TnH}$ & 14.1 & 14.0 & - & - & $-161.3 / 66.8$ & $-157.7 / 67.9$ & - & - \\
\hline
\end{tabular}

